\title{
STABILITY PHENOMENA FOR RESONANCE ARRANGEMENTS
}

\author{
NICHOLAS PROUDFOOT AND ERIC RAMOS
}

(Communicated by Patricia L. Hersh)

\begin{abstract}
We prove that the $i^{\text {th }}$ graded pieces of the Orlik-Solomon algebras or Cordovil algebras of resonance arrangements form a finitely generated FS ${ }^{\mathrm{op}}$ module, thus obtaining information about the growth of their dimensions and restrictions on the irreducible representations of symmetric groups that they contain.
\end{abstract}

\section{INTRODUCTION}

Let $\mathcal{A}(n)$ be the collection of all hyperplanes in $\mathbb{R}^{n}$ that are perpendicular to some nonzero vector with entries in the set $\{0,1\}$. This hyperplane arrangement is called the resonance arrangement of rank $n$. The resonance arrangement has connections to algebraic geometry, representation theory, geometric topology, mathematical physics, and economics; for a survey of these connections, see [4, Section 1]. Of particular interest is the set of chambers of $\mathcal{A}(n)$. Amazingly, despite the simplicity of the definition, no formula for the number of chambers as a function of $n$ is known. A more refined invariant of $\mathcal{A}(n)$ is its characteristic polynomial, whose coefficients (after taking absolute values) have sum equal to the number of chambers. Kühne has made some progress toward understanding the coefficient of $t^{n-i}$ in the characteristic polynomial as a function of $n$ with $i$ fixed. Our purpose is to shed a new light on Kühne's result, to generalize it to a wider class of arrangements, and to study the action of the symmetric group $\Sigma_{n}$ on various algebraic invariants of these arrangements.

Let $S \subset \mathbb{R}$ be any finite set, and let $\mathcal{A}_{S}(n)$ be the collection of hyperplanes that are perpendicular to a nonzero vector with entries in $S$. If $S=\{0,1\}, \mathcal{A}_{S}(n)$ is the resonance arrangement. If $S=\{ \pm 1\}$, it is the threshold arrangement, which is studied in [3]. For each positive integer $d$, let $M_{S}(n, d)$ denote the set of $n$-tuples of vectors in $\mathbb{R}^{d}$ such that no nontrivia 1 linear combination of all $n$ vectors with coefficients in $S$ is equal to zero. The cohomology ring of $M_{S}(n, d)$ is generated in degree $d-1$ [2, Corollary 5.6]. If $d$ is even, the presentation of this ring in [2] coincides with that of the Orlik-Solomon algebra of $\mathcal{A}_{S}(n)$ (with all degrees multiplied by $d-1$ ) [6]. If $d$ is odd and greater than 1 , then it coincides with that of the Cordovil algebra of $\mathcal{A}_{S}(n)$ (with all degrees multiplied by $d-1$ ) [1]; see

Received by the editors November 2, 2020, and, in revised form, November 18, 2020.

2020 Mathematics Subject Classification. Primary 14N20.

The first author was supported in part by NSF grants DMS-1565036 and DMS-1954050. The second author was supported in part by NSF grant DMS-1704811.

${ }^{1}$ Nontrivial means that, if $0 \in S$, we do not allow all coefficients to be 0 . 
also [5, Example 5.8] 2 In particular, for any $n \geq 1, d \geq 2$, and $i \geq 0$, the dimension $b_{S}^{i}(n)=\operatorname{dim} H^{(d-1) i}\left(M_{S}(n, d) ; \mathbb{Q}\right)$ is equal to $(-1)^{i}$ times the coefficient of $t^{n-i}$ in the characteristic polynomial of $\mathcal{A}_{S}(n)$.

These vector spaces carry more information than just their dimension; they also carry actions of the symmetric group $\Sigma_{n}$, which acts by permuting the $n$ vectors. These representations are isomorphic for all even $d \geq 2$ and for all odd $d \geq 3$, but the $d=2$ and $d=3$ cases are genuinely different. The total cohomology $H^{*}\left(M_{S}(n, 3) ; \mathbb{Q}\right)$ with all degrees combined is isomorphic as a representation of $\Sigma_{n}$ to $H^{0}\left(M_{S}(n, 1) ; \mathbb{Q}\right)$, which is the permutation representation with basis indexed by the chambers of $\mathcal{A}_{S}(n)$ [5, Theorem 1.4(b)].

For fixed $S \subset \mathbb{R}, d \geq 2$, and $i \geq 0$, we will define in the next section a contravariant module $B_{S}^{i, d}$ over the category of finite sets with surjections that takes the set $[n]$ to $H^{(d-1) i}\left(M_{S}(n, d) ; \mathbb{Q}\right)$.

Theorem 1.1. The module $B_{S}^{i, d}$ is finitely generated in degrees $\leq|S|^{i}$.

Combining Theorem 1.1 with [7, Theorem 4.1], we obtain the following numerical results 3

Corollary 1.2. Fix a finite set $S \subset \mathbb{R}$ and a pair of integers $d \geq 2$ and $i \geq 0$.

(1) The generating function

$$
G_{S}^{i}(t):=\sum_{n=1}^{\infty} b_{S}^{i}(n) t^{n}
$$

is a rational function with poles contained in the set $\left\{1 /\left.j|1 \leq j \leq| S\right|^{i}\right\}$, with at worst a simple pole at $|S|^{-i}$. Equivalently, there exist polynomials $\left\{\left.c_{S}^{i, j}(n)|1 \leq j \leq| S\right|^{i}\right\}$ such that, for $n$ sufficiently large,

$$
b_{S}^{i}(n)=\sum_{j=1}^{|S|^{i}} c_{S}^{i, j}(n) j^{n},
$$

and the last polynomial $c_{S}^{i,|S|^{i}}(n)$ is a constant polynomial.

(2) For any partition $\lambda$ of $n$, let $V_{\lambda}$ denote the irreducible representation of $\Sigma_{n}$ indexed by $\lambda$. If $\operatorname{Hom}_{\Sigma_{n}}\left(V_{\lambda}, H^{(d-1) i}\left(M_{S}(n, d) ; \mathbb{Q}\right)\right) \neq 0$, then $\lambda$ has at most $|S|^{i}$ rows.

(3) For any partition $\lambda$ with $n \geq|\lambda|+\lambda_{1}$, let $\lambda(n)$ be the padded partition of $n$ obtained from $\lambda$ by adding a row of length $n-|\lambda|$. For any $\lambda$, the function

$$
n \mapsto \operatorname{dim} \operatorname{Hom}_{\Sigma_{n}}\left(V_{\lambda(n)}, H^{(d-1) i}\left(M_{S}(n, d) ; \mathbb{Q}\right)\right)
$$

is bounded above by a polynomial in $n$. In particular, if $\lambda$ is the empty partition, this says that the multiplicity of the trivial representation in $H^{(d-1) i}\left(M_{S}(n, d) ; \mathbb{Q}\right)$ is bounded above by a polynomial in $n$.

\footnotetext{
${ }^{2}$ For $d$ odd, the presentation in [2] incorrectly omits the relations that each of the generators squares to zero.

${ }^{3}$ The deepest of these statements, namely the fact that the dimension generating function for

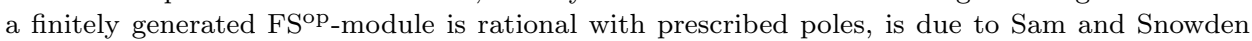
[8. Corollary 8.1.4].
} 
Remark 1.3. A stronger version of item (1) above for the resonance arrangement appears in 4, Theorem 1.4]. Kühne proves that the polynomials $c_{\{0,1\}}^{i, j}(n)$ are all constant (i.e. that all poles of $G_{\{0,1\}}^{i}(t)$ are simple), obtains bounds on their sizes, and shows that the equality holds for all $n$, not just sufficiently large $n$ (i.e. that the limit as $t$ goes to $\infty$ of $G_{\{0,1\}}^{i}(t)$ is zero). It should be possible to categorify Kühne's theorem by proving that the restriction of $B_{\{0,1\}}^{i, d}$ to the category of ordered surjections [8] is isomorphic to a direct sum of shifts of principal projectives, with summands indexed by Kühne's functional prototypes. The cost of working with ordered surjections would be that we would lose all information about the action of the symmetric group.

\section{THE PROOF}

Let FS denote the category whose objects are nonempty finite sets and whose morphisms are surjective maps. An $\mathbf{F S}^{\mathbf{o p}}$-module over $\mathbb{Q}$ is a contravariant functor from FS to the category of rational vector spaces. For each finite set $F$, we have the principal projective module $P_{F}$, which sends a finite set $E$ to the vector space with basis $\operatorname{Hom}_{\mathrm{FS}}(E, F)$, with morphisms defined on basis elements by composition. An $\mathrm{FS}^{\mathrm{op}}$-module $N$ is said to be finitely generated if it is a quotient of a finite sum $\oplus_{i} P_{F_{i}}$ of principal projectives, and it is said to be finitely generated in degrees $\leq \boldsymbol{m}$ if the sets $F_{i}$ can all be taken to have cardinality less than or equal to $m$. This is equivalent to saying that, for all $E$, the vector space $N(E)$ finite dimensional and is spanned by the images of the pullbacks along various maps $\varphi: E \rightarrow F$, where $F$ has cardinality less than or equal to $m$.

Lemma 2.1. Suppose that $N_{1}$ is finitely generated in degrees $\leq m_{1}$ and $N_{2}$ is finitely generated in degrees $\leq m_{2}$. Then the pointwise tensor product $N_{1} \otimes N_{2}$ is finitely generated in degrees $\leq m_{1} m_{2}$.

Proof. We immediately reduce to the case where $N_{1}=P_{\left[m_{1}\right]}$ and $N_{2}=P_{\left[m_{2}\right]}$. For any $\varphi: E \rightarrow[m]$, let $e_{\varphi}$ denote the corresponding basis element of $P_{[m]}(E)$. Then $N_{1} \otimes N_{2}$ has basis

$$
\left\{e_{\varphi_{1}} \otimes e_{\varphi_{2}} \mid \varphi_{1}: E \rightarrow\left[m_{1}\right], \varphi_{2}: E \rightarrow\left[m_{2}\right]\right\} .
$$

Given the pair of surjections $\left(\varphi_{1}, \varphi_{2}\right)$, let $F \subset\left[m_{1}\right] \times\left[m_{2}\right]$ denote the image of $\varphi_{1} \times \varphi_{2}$, let $\varphi=\varphi_{1} \times \varphi_{2} \in \operatorname{Hom}_{\mathrm{FS}}(E, F)$, and let $\psi_{1}: F \rightarrow\left[m_{1}\right]$ and $\psi_{2}: F \rightarrow\left[m_{2}\right]$ denote the coordinate projections. It is clear that we have $e_{\varphi_{1}} \otimes e_{\varphi_{2}}=\varphi^{*}\left(e_{\psi_{1}} \otimes e_{\psi_{2}}\right)$. Since the cardinality of $F$ is at most $m_{1} m_{2}$, this completes the proof.

Fix a positive integer $d$ and a finite set $S \subset \mathbb{R}$. To any finite set $E$, we associated the space $M_{S}(E, d)$ of $E$-tuples of vectors in $\mathbb{R}^{d}$ such that any nontrivial linear combination of the vectors with coefficients in $S$ is nonzero. Given a surjection $\varphi: E \rightarrow F$, we obtain a map

$$
\varphi_{*}: M_{S}(E, d) \rightarrow M_{S}(F, d)
$$

by adding the vectors in each fiber of $\varphi$. These maps define a functor from FS to the category of topological spaces. By taking rational cohomology in degree $(d-1) i$, we obtain an $\mathrm{FS}^{\mathrm{op}}$-module $B_{S}^{i, d}$. We prove the following theorem, which implies the three statements in the introduction. 
Proof of Theorem 1.1. As noted above, the cohomology of $M_{S}(E, d)$ is generated as an algebra in degree $d-1$, hence $B_{S}^{i, d}$ is a quotient of $\left(B_{S}^{1, d}\right)^{\otimes i}$. By Lemma 2.1. this means that it is sufficient to prove that $B_{S}^{1, d}$ is finitely generated in degrees $\leq|S|$. For any finite set $F$, the vector space $B_{S}^{1, d}(F)$ has a generating set indexed by nonzero elements of $S^{F}$ [2, Corollary 5.6] (these generators form a basis unless two nonzero elements of $S^{F}$ are proportional, in which case the corresponding generators are equal). For any nonzero $v \in S^{F}$, let $x_{v} \in B_{S}^{1, d}(F)$ be the corresponding generator. Concretely, if we take $x \in H^{d-1}\left(\mathbb{R}^{d} \backslash\{0\} ; \mathbb{Q}\right)$ to be the standard generator, then $x_{v}$ is equal to the pullback of $x$ along the map

$$
f_{v}: M_{S}(F, d) \rightarrow \mathbb{R}^{d} \backslash\{0\}
$$

that sends an $F$-tuple of vectors to its linear combination with coefficients determined by $v$. Given a surjection $\varphi: E \rightarrow F$, we have $f_{v} \circ \varphi_{*}=f_{\varphi^{*} v}$, and therefore

$$
\varphi^{*}\left(x_{v}\right)=\varphi^{*} \circ f_{v}^{*}(x)=f_{\varphi^{*} v}^{*}(x)=x_{\varphi^{*} v} \in B_{S}^{1, d}(E) .
$$

Since every element of $S^{E}$ may be pulled back from a subset of cardinality at most $|S|, B_{S}^{1, d}$ is generated in degrees $\leq|S|$.

Remark 2.2. Our construction also works if we replace $\mathbb{R}$ with an arbitrary field $k$ and we take $S$ to be a finite subset of $k$. We define the arrangement $\mathcal{A}_{k, S}(n)$ in $k^{n}$ as above, we denote its complement by $M_{k, S}(E, 1)$, and we take $B_{k, S}^{i, 1}(E)$ to be the étale cohomology group $H_{\text {ét }}^{i}\left(M_{k, S}(E, 1) \otimes_{k} \bar{k} ; \mathbb{Q}_{l}\right)$ for some prime $l$ not equal to the characteristic of $k$, which is isomorphic to the degree $i$ part of the Orlik-Solomon

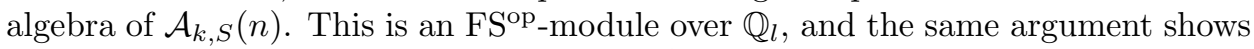
that it is finitely generated in degrees $\leq|S|^{i}$.

An interesting special case is where $k=\mathbb{F}_{q}$ is a finite field and $S=k$, so that our arrangement $\mathcal{A}_{\mathbb{F}_{q}, \mathbb{F}_{q}}(n)$ is the collection of all hyperplanes in $\mathbb{F}_{q}^{n}$. This arrangement has characteristic polynomial $(t-1)(t-q) \cdots\left(t-q^{n-1}\right)$, and therefore the $i^{\text {th }}$ Betti number is equal to the evaluation of the $i^{\text {th }}$ elementary symmetric polynomial at the values $1, q, \ldots, q^{n-1}$. This implies that the Hilbert series of our module is

$$
q^{\left(\begin{array}{l}
i \\
2
\end{array}\right)} t^{i} \prod_{j=0}^{i} \frac{1}{1-q^{j} t},
$$

which has simple poles at $q^{-j}$ for $j=0,1, \ldots, i$. The projectivization of $M_{\mathbb{F}_{q}, \mathbb{F}_{q}}(n, 1)$ $\otimes_{\mathbb{F}_{q}} \overline{\mathbb{F}}_{q}$ is a Deligne-Lusztig variety for the group $\mathrm{GL}_{n}\left(\mathbb{F}_{q}\right)$.

\section{ACKNOWLEDGMENT}

The authors are grateful to Lou Billera for telling them about the arrangement $\mathcal{A}(n)$ and about Kühne's work.

\section{REFERENCES}

[1] R. Cordovil, A commutative algebra for oriented matroids: Geometric combinatorics (San Francisco, CA/Davis, CA, 2000), Discrete Comput. Geom. 27 (2002), no. 1, 73-84, DOI 10.1007/s00454-001-0053-8. MR.1871690

[2] Mark de Longueville and Carsten A. Schultz, The cohomology rings of complements of subspace arrangements, Math. Ann. 319 (2001), no. 4, 625-646, DOI 10.1007/PL00004452. MR.1825401

[3] Samuel C. Gutekunst, Karola Mészáros, and T. Kyle Petersen, Root cones and the resonance arrangement, Electron. J. Combin. 28 (2021), no. 1, Paper No. 1.12, 39, DOI 10.37236/8759. $\operatorname{MR} 4245245$ 
[4] Lucas Kühne, The universality of the resonance arrangement and its Betti numbers, arXiv:2008.10553

[5] Daniel Moseley, Equivariant cohomology and the Varchenko-Gelfand filtration, J. Algebra 472 (2017), 95-114, DOI 10.1016/j.jalgebra.2016.10.010. MR3584871

[6] Peter Orlik and Louis Solomon, Combinatorics and topology of complements of hyperplanes, Invent. Math. 56 (1980), no. 2, 167-189, DOI 10.1007/BF01392549. MR558866

[7] Nicholas Proudfoot and Ben Young, Configuration spaces, FS ${ }^{\circ}$-modules, and KazhdanLusztig polynomials of braid matroids, New York J. Math. 23 (2017), 813-832. MR3690232

[8] Steven V. Sam and Andrew Snowden, Gröbner methods for representations of combinatorial categories, J. Amer. Math. Soc. 30 (2017), no. 1, 159-203, DOI 10.1090/jams/859. MR3556290

Department of Mathematics, University of Oregon, Eugene, Oregon 97403

Email address: njp@uoregon.edu

Department of Mathematics, Bowdoin College, Brunswick, Maine 04011

Email address: e.ramos@bowdoin.edu 